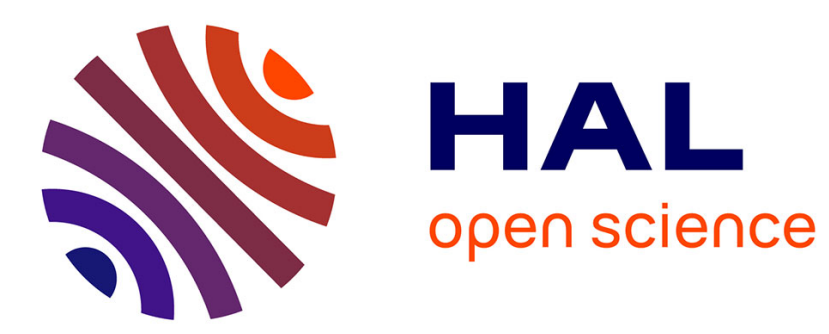

\title{
A few key theoretical issues of importance in modern molecular electrochemistry
}

Alexander Oleinick, Irina Svir, Christian Amatore

\section{To cite this version:}

Alexander Oleinick, Irina Svir, Christian Amatore. A few key theoretical issues of importance in modern molecular electrochemistry. Current Opinion in Electrochemistry, 2019, 13, pp.33-39. 10.1016/j.coelec.2018.10.008 . hal-02338073

\section{HAL Id: hal-02338073 https://hal.science/hal-02338073}

Submitted on 29 Oct 2019

HAL is a multi-disciplinary open access archive for the deposit and dissemination of scientific research documents, whether they are published or not. The documents may come from teaching and research institutions in France or abroad, or from public or private research centers.
L'archive ouverte pluridisciplinaire HAL, est destinée au dépôt et à la diffusion de documents scientifiques de niveau recherche, publiés ou non, émanant des établissements d'enseignement et de recherche français ou étrangers, des laboratoires publics ou privés. 


\title{
Opinion article
}

\section{A few key theoretical issues of importance in modern molecular electrochemistry}

\author{
Alexander Oleinick, ${ }^{1}$ Irina Svir ${ }^{1}$ and Christian Amatore ${ }^{1,2, *}$
}

\author{
Addresses \\ ${ }^{1}$ PASTEUR, Département de chimie, École normale supérieure, PSL University, Sorbonne Université, \\ CNRS, 75005 Paris, France \\ ${ }^{2}$ State Key Laboratory of Physical Chemistry of Solid Surfaces, College of Chemistry and Chemical \\ Engineering, Xiamen University, Xiamen, 361005, China \\ * Corresponding author: Amatore, Christian (christian.amatore@ens.fr)
}

This opinion paper details three typical cases in which new theoretical concepts need to be implemented in molecular electrochemistry in order to rationalize experimental results obtained in nanoscale cells or performed in new electrolytic media such as RTILs.

Keywords: Theory at nanoscale; Nanoelectrochemistry; Single molecule electrochemistry; RTILs; Numerical simulation.

\section{Introduction}

The advent of nanoscale electrochemistry has opened a multitude of problems that could only glimpsed at ca 25 years ago when microelectrodes started to make their way in laboratories for routine experiments [1]. At that time the main theoretical issues seemed mostly be related to the difficulty of treating edge effects under steady and quasi-steady state electrochemical conditions, and to the consequences of having diffusion layers and interfacial diffuse layers merging to the point where electroneutrality cannot be considered anymore to apply over the solution range where concentration gradients of electroactive species develop [2-4]. These problems still keep their important topicality, but many others have been arising that deal more directly with the nano-sizes of electrodes and with the important role played by nanoscale structures forming in electrolytes near electrodes.

The format allowed for this type of perspective does not permit discussing all such theoretical problems even assuming that we could foresee all of them at the present time. So we decided to select three topics that have currently high experimental and technological interest and in which these problems are playing significant roles though it seems to us that this is not so well perceived by experimentalists.

\section{Single molecule electrochemistry in nano-confined dual-electrodes cells}

The so-called single molecule electrochemistry $[5,6]$ term was coined more than 20 years ago. The method is taking advantage of the current amplification created when a feedback mode is 
established between two close electrodes, e.g., a SECM tip and a conducting substrate [7]. In fact this elaborates on the well-known principle of redox cycling between two close electrodes [8], except that the use of nanometric electrodes provides an excellent signal-to-noise ratio allowing the precise measurement of currents ranging from a few picoamperes to a few femtoamperes due to the redox-cycling amplification of only one (to a few) molecules "trapped" within nanometer-sized thin-layer cells formed by nanometric electrodes separated by nanometric gaps $[5,9]$. The possibility of monitoring electrochemically such small numbers of molecules has stimulated a huge interest in expectation of observing and analyzing for the first time fluctuations in electrochemical properties (see a recent review discussing this in deeper depth than here) [10]. Indeed, classical electrochemical experiments give access only to statistically averaged values because of the huge numbers of events involved in each single measurement $[11,12]$. New nanofabrication methods have been recently developed to create nanochannels with interelectrodes gaps of precise dimensions in the tens of nanometers range in hope to evaluate in more details the stochastic properties of a redox molecules [12-15]. More interestingly in view of the subject of this perspective is the fact that such nano-gap electrochemical cells allowed investigating the effect of diffuse layer electrical fields on the meaning of electron transfer (ET) kinetics [16-21], as well as the consequences of adsorption of one member of the investigated redox couple [22], while these effects are difficultly interpreted when observed under classical electrochemical conditions.

However, to the best of our knowledge, none of these studies or many others reported on the topics of single entities electrochemistry $[10,23]$ have considered a point that seems to us to have an extreme importance, maybe because one thinks to such events as one is used to do when analyzing classical electrochemical experiments. Indeed, under classical electrochemistry the continuity of the current pathway between the working and auxiliary electrodes is ensured by a sequence of different phenomena that occur almost simultaneously (i.e., within the limit of the speed of light across the electrochemical cell) and that are generally represented as in Figure $1 \mathrm{~A}$. The use of the reference electrode allows uncoupling the two active electrodes by relating the current flow across the cell to the potential applied to the working electrode independently of that applied to the auxiliary electrode. However the current intensity flowing through the working electrode must also flow through the auxiliary electrode in order to complete the whole electrical circuit. In other words, one takes advantage that classical electrochemical cells contains a colossal number of redox active molecules (in fact a not so small fraction of the Avogadro number) to ensure that within the duration of an electron transfer event occurring at the working electrode, viz., a few femtoseconds, another one occurs in the opposite direction at the auxiliary electrode. This only enables the ionic current flowing through the solution bulk to be converted into the electronic current that flows through the electrodes and the external equipment. On the other hand this also maintain the global electroneutrality of the bulk solution. Evidently, this cannot occur so easily when the number of molecules contained in a nanoelectrochemical cell becomes extremely small. Pushed to the extreme, in a zeptoliter volume containing a single ferrocene 
molecule, the one-electron oxidation at the working anode cannot be compensated by a simultaneous one-electron reduction occurring at the auxiliary cathode whenever the potential drop across the cell is maintained over the range of the ferrocene/ferricinium wave. In other words, upon oxidizing the single ferrocene molecule contained in the nano-cell one has created a charge that cannot be immediately compensated by a Faradic process and drastically increases the potential of the formerly electroneutral zeptoliter solution volume [24].

Figure 1

A. "Classical" electrochemistry in usual cell

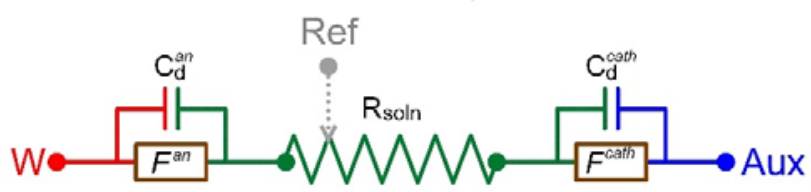

B. "Single Molecule" electrochemistry in nanocell

B-1. Anodic current flow

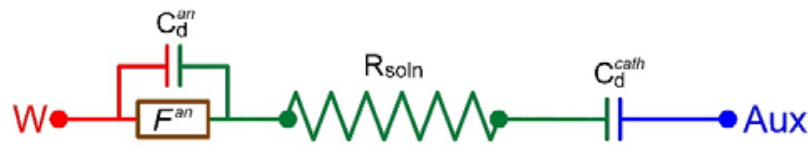

\section{B-2. Cathodic current flow}

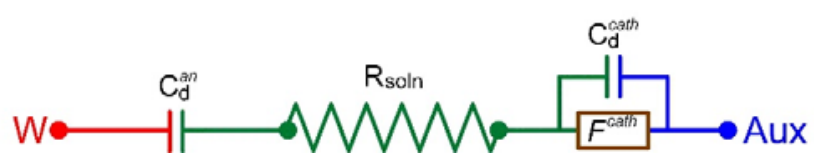

Comparison between the functioning modes of an electrochemical cell under (A) classical conditions for two- or three-electrode operating mode or (B) during "single molecule" operation (two-electrode mode) in a zeptoliter volume cell containing a single redox molecule. The color code indicates the nature of the current flow: red = electronic current towards the working electrode lead (W); blue = electronic current from the auxiliary electrode lead (Aux); green = ionic current in the solution; brown = Faradic impedances. Each double layer capacitor "couples" an electronic contribution to an ionic one of same magnitude (charge/discharge of the capacitance); each Faradic impedance "converts" an electronic current into an ionic one or reciprocally hence the naming "anodic" (B1) or "cathodic" (B2) current flows.

Nonetheless, experimentally $[13,25]$, one observes that this is associated to an anti-correlated current flowing at the cathode. This evidences that under such circumstances the electrical circuit 
is closed by a non-Faradic mean, viz., through charging of the cathode double layer (Figure 1B-1) due to the increase of the electrolyte potential, a known phenomenon usually named "capacitive coupling" [26]. The opposite occurs when the ensuing ferricinium ion reaches the cathode where it is reduced as is sketched in Figure 1B-2, thus relaxing the solution potential increase. In fact, note that by construction, except if a proper accounting of the successive events is made in experiments such as those in Refs [12-15], it is difficult to decide at which electrode an electron transfer is occurring and is compensated by a mirror capacitive current at the other one.

Presumably, this is the source of the uncommon effects related to the composition of the electrolytes cited above [16-21]. We therefore strongly believe that this problem of single molecule electrochemistry should be precisely investigated theoretically before any hope of using these approaches to unravel electrochemical properties beyond investigations related to the stochastic nature of fundamental electrochemical laws $[11,12,27]$. The main difficulties in building serious theoretical approaches to the problem is that one is dealing here with a succession of stochastic single events, each one having its own specific features. Hence, no classical formulations such as those implicitly considered for each component in the schematic diagrams in Figure 1 can be used. Brownian molecular dynamics may be useful in this context [24] for transport including migration in the double layers but will require its coupling with DFT (QM/MM) to treat molecular organization at the interfaces [28] as well as electron transfer kinetics and thermodynamics $[29,30]$ since again no classical electron transfer theory such as Marcus' or Nernst's can be used. In our opinion the availability of theoretical contributions on these subjects will be useful not only for fundamental investigations and basic knowledge but also for many practical issues that arise presently in many advances for electrocatalysis in nanoconfined environments, energy conversion and storage in supercapacitors.

\section{Electrochemistry in room temperature ionic liquids (RTILs)}

This is a second example of a rather recent experimental electrochemical field that has rapidly expanded owing to the peculiar physical and chemical properties of RTILs and their importance in electrochemistry [31-33]. However, it is clear that the very structure of RTILs [34] imposes that all classical electrochemical concepts need to be revisited and cannot be applied in these media and carry the same meaning as under classical electrochemical conditions. Indeed, almost all the concepts we use on everyday basis to analyze electrochemical data have been developed for simple electrolytes diluted in non-viscous solvent media. Hence, their very roots appear fully irrelevant to situations in which the medium itself is composed of organic bulky ions in close contact [35] in which high degrees of order are maintained over mesoscopic spatial scales [36].

For example, the very structure and dynamics of electrode/RTIL [37] or water/RTIL [38] electrified interfaces have been investigated appears to contradict predictions based on the classical GouyChapman-Stern theory. This questions in turn the very notion of reorganization energies and work terms whose roles in ET theories are critical. Similarly, what precise phenomena underlie the experimental values of diffusion coefficients measured in RTILs and justify their Arrhenius-type 
temperature dependence [39]? All these issues are critical as soon as one wants to use and rationalize electrochemical data [40] in such media. It seems to us that owing to the critical roles of the nanoscale properties (of importance for conditioning local structures as well as for dynamics) that are concerned, any classical modelling based on usual physicochemical laws will not provide any suitable access to understand the real fundamental nature of the parameters involved in the simulations. To be more precise, even if experiments supported by precise simulations based on classical electrochemical laws [41] provide values for the above parameters, such theoretical approaches alone will never be able to rationalize the origin of their distinctiveness compared to their analogs under usual electrochemical conditions.

However, owing to the increasing involvement of RTILs in many nanoscale-electrochemical topics such as advanced supercapacitors we believe that a profound understanding of the fundamental phenomena that govern their peculiar behavior is required. In this respect, considering the important role of nanoscale structures and that of the dynamics of their local deformations, we believe that molecular dynamics simulations will certainly provide the needed understanding. Indeed, these approaches have already shown their ability in investigating structural and dynamics problems involved in many RTILs [34,35] in particular by providing important information on ion pairing and ion cages structures and life-times, i.e., about fundamental issues of interest for understanding electrochemistry in these media. Hence, multiscale theoretical approaches involving molecular dynamics coupled to DFT (QM/MM) [28-30] should provide interesting outcomes to unravel the physicochemical phenomena that underlie electrochemistry in RTILs; see e.g. references [37-43] for recent interesting approaches in such directions.

\section{Simulations of transient voltammetry involving realistic ohmic and capacitive contributions and dynamic adsorption of reactive electroactive species}

Transient voltammetry has proven its great advantages over most electrochemical methods for unravelling complex mechanistic features in electrochemistry. Yet, under most circumstances, viz., as soon as the electrochemical mechanism is not straightforward, one cannot rely on the application of analytical relationships between peak potentials or current intensities with scan rates, etc. [44], to characterize the mechanism and extract the relevant kinetic and thermodynamic information. Then a complete theoretical analysis of the voltammetric data based on comparison with simulations is required. Today several user-friendly commercial programs allow simulations of $1 \mathrm{D}$ and $2 \mathrm{D}$ electrochemical problems involving complex mechanistic sequences in solution (e.g., among the most popular ones: $\operatorname{DigiSim}^{\odot}$ [45], DigiElec ${ }^{\odot}$ [46], KISSA ${ }^{\odot}$ [47-49]) and this can be extended by self-programming of multi-purpose programs originally devised for physical problems such as $\mathrm{Comsol}^{\odot}$. Hence, one may have the impression that the efforts started in the middle of the sixties have reached their final goal.

However, today, many problems at hand in molecular electrochemistry are no more limited to situations where the only role of the electrode was to provide or accept electrons while the 
ensuing chemistry took place in solution. Indeed, many electrocatalytic systems investigated nowadays involve critical dynamic interplays between solution and surface kinetic intermediates. This in turn involve dynamic adsorption/desorption steps and leads to competitive, possibly parallel, reactions of intermediates on the electrode surface and in the solution [50,51]. Figure $2 \mathrm{~A}$ represents the general situation expected for a simple reduction pathway in which electron transfers may occur either in solution at the OHP or between adsorbed species of the same redox couple.

This general scheme is contrasted in Figure $2 \mathrm{~B}$ to that of a solution electron transfer according to the classical Smoluchowski-Debye model. This comparison evidences that interfacial ET may well also occur when the substrate is not at the OHP. Indeed, transposition of the SmoluchowskiDebye equation for rate constants evidences that long range ET may occur. Evidently since in usual media ET rate constant decays exponentially with the distance this is irrelevant at macroor even microelectrodes since anyway the uncertainty on the local ET position does not matter (except for the magnitude of the corresponding rate constant) because the dimension of diffusion layers is excessively large compared to a few angstroms uncertainty on the exact ET positions. This is simply reflected by the extracted ET rate constant value. However, this may become a serious problem at nanoelectrodes or at catalytic nanoparticles [52]. In this case simulations ought to consider stochastic probabilities accounting for the fact that ETs may occur from/to a point located significantly deep in the diffusion layer that would be predicted on the basis of Fick's laws. This may be extremely important when the nanoelectrode or nanoparticle size are small enough for the distinction between the diffuse layer and the diffusion one to become ambiguous $[2-4,20]$ or when partial blocking of the electrode is associated to the electrochemical process [22,53-56]. Note that this involves a different kind of stochastic problem than those considered in the case of single molecule electrochemistry.

To conclude this section we want to discuss two linked major problems in voltammetric experiments especially when compared with simulations. This concerns the effect of ohmic drop and time constant (or the way of correcting the distortions they induce [57]). Indeed, to simulate the effect of ohmic drop and time constant, commercial simulation programs such as DigiSim ${ }^{\odot}$ or DigiElec $^{\odot}$ consider a classical $R C$ series circuit where $C$ is a pure capacitive element featuring the double layer capacitance acting in parallel to the Faradic impedance and $R$ represents the uncompensated solution resistance, i.e., that between the working and reference electrodes (see Figure $1 \mathrm{~A}$ ). However, it is enough to consult the voltammetric literature to understand that, except for a few exploited cases, the assumption of a constant double layer capacitance over a potential range suited to voltammetric investigations is a myth, even when the voltammetric range is set sufficiently far away from the point of zero-charge. This is the main reason why such pseudo-corrections have not been implemented in $\mathrm{KISSA}^{\odot}$.

In fact under most experimental conditions the double layer capacitance is best represented by a constant phase element (CPE) [58,59]. Though CPE double layer capacitances are seldom considered in rationalizing voltammetric experiments, CPEs are now widely accepted and used to 
model electrical equivalent circuits in electrochemical impedance spectroscopy [60]. Incorrectly predicted ohmic drop and capacitive background may have severe consequences on the interpretation of transient voltammetric data as soon precise measurements are sought [58]. This clearly claims for the integration of CPEs in finite difference numerical simulation programs able to treat any complex mechanistic sequence.

\section{Figure 2}

A. General electrochemical reduction pathway

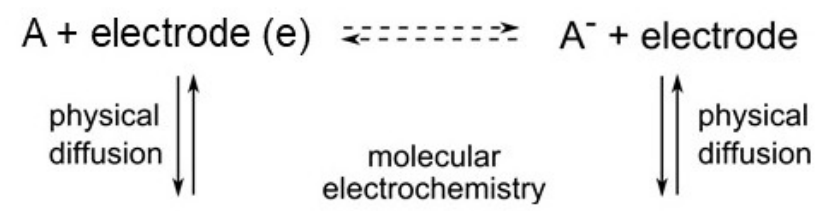
$\mathrm{A}, \mathrm{OHP}+$ electrode $(\mathrm{e})$

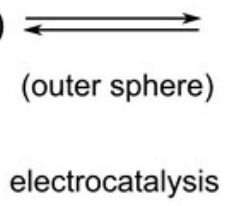
$\mathrm{A}^{-}, \mathrm{OHP}$
adsorption $\mid \uparrow$
electrocatalysis

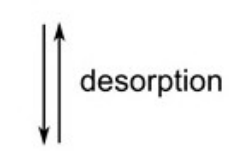

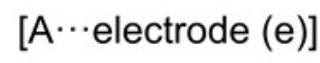
(inner sphere)

B. General chemical reaction pathway

(Smoluchowski-Debye model)

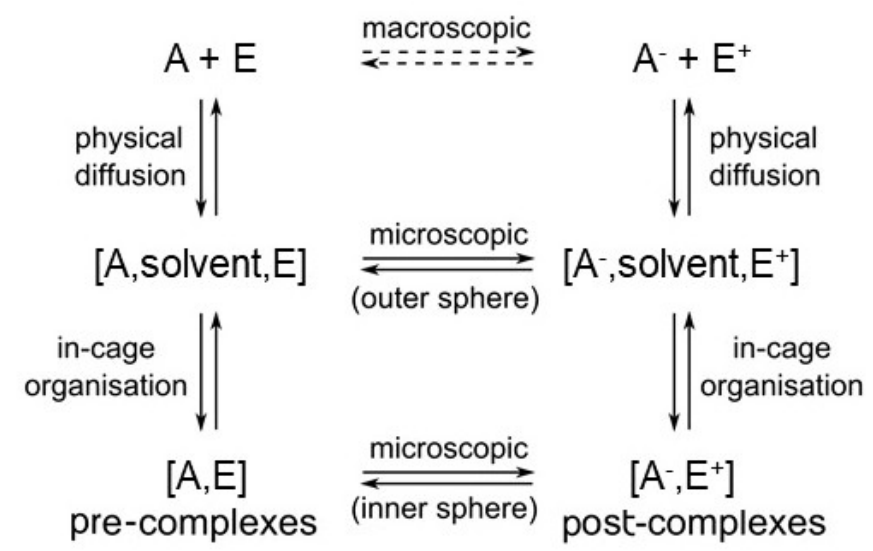

(A) General mechanism for the reversible 1-e reduction of species A into $B$ involving two competitive pathways: electron transfer occurring in solution at the OHP, vs. electron transfer between adsorbed species. (B) Comparison to the general pathway for electron transfer in solution according to Smoluchowski-Debye model. (Adapted from reference 51). 


\section{Conclusion}

The increasing availability of nanoscale technologies or of new electrolytes has allowed precise and accurate experimental investigations of electrochemical systems under conditions that would have been unthinkable two decades ago. However, these experiments are most often rationalized using theoretical concepts that were invented and refined for making sense of situations corresponding to much larger time and space scales and involving much larger numbers of molecules or ions. Ironically, the outcomes of such discordant views produces results that look perfectly adequate, at least up to the moment one starts to interrogate the meaning of the values extracted for the usual electrochemical parameters. In this short opinion paper we illustrated three typical cases in which, despite the huge number of published experimental data, new theoretical concepts need to be implemented.

\section{Acknowledgments}

This work was supported in parts by CNRS, ENS, Sorbonne University and PSL University (UMR 8640 PASTEUR). CA acknowledges Xiamen University for his position of Distinguished Professor.

\section{References}

1 Amatore C: Electrochemistry at Ultramicroelectrodes. "Physical Electrochemistry: Principles, Methods and Applications" (I. Rubinstein, Ed.), M. Dekker, New York. 1995, Chap.4: 131-208.

$2^{\circ} \quad$ Amatore C, Lefrou C: Is Cyclic Voltammetry Above A Few Hundred KiloVolts per Second Still Cyclic Voltammetry? J. Electroanal. Chem. 1990, 296: 335-358.

$3^{\circ} \quad$ Norton JD, White HS, Feldberg SW: Effect of the electrical double layer on voltammetry at microelectrodes. J. Phys. Chem. 1990, 94:6772-6780.

- These two papers explored for the first time the consequences of decreasing diffusion layers thicknesses down to the size of double layers (reference 2 considers nanosecond electrochemistry at microelectrodes while reference 3 envision the case of steady state voltammetry at nano-sized electrodes).

4 Smith CP, White HS: Theory of the voltammetric response of electrodes of submicron dimensions violation of electroneutrality in the presence of excess supporting electrolyte. Anal. Chem. 1993, 65:3343-3353.

$5^{\circ \bullet} \quad$ Fan FRF, Bard A: Electrochemical detection of single molecules. Science 1995, 267:871874.

- This paper opened the way to single entities electrochemistry.

6 Fan FRF, Kwak J, Bard AJ: Single Molecule Electrochemistry. J. Am. Chem. Soc. 1996, 118: 9669-9675.

7 Bard AJ, Mirkin MV, Unwin PR, Wipf DO: Scanning electrochemical microscopy. 12. Theory and experiment of the feedback mode with finite heterogeneous electron-transfer kinetics and arbitrary substrate size . J. Phys. Chem. 1992, 96:1861-1868 . 
8 Anderson LB, Reilley CN: Thin-layer electrochemistry: steady-state methods of studying rate processes. J. Electroanal. Chem. 1965, 10:295-305.

$9^{\circ \bullet}$ Sun P, Mirkin MV: Electrochemistry of individual molecules in zeptoliter volumes. J. Am. Chem. Soc. 2008, 130: 8241-8250.

- To the best of our knowledge this paper provides for the first time measurements of individual molecules in a zeptoliter electrochemical cell of defined dimensions (see references [13-15,22] for more elaborate approaches made feasible by the development of nanofabrications).

$10^{\circ}$ White HS, McKelvey K: Redox cycling in nanogap electrochemical cells. Current Opinion in Electrochemistry 2017, $7: 48-53$.

- This review exposes the state of the art in electrochemistry at nanogap electrochemical cells.

$11^{\circ \bullet}$ Amatore C, Grün F, Maisonhaute E: Electrochemistry within a limited number of molecules: delineating the fringe between stochastic and statistical behavior. Angew. Chem. Int. Edn. 2003, 42: 4944-4947.

- This paper introduced the necessity of considering stochastic limitations in electrochemistry of a finite number of redox active molecules.

$12^{\circ}$ Singh P, Lemay SG: Stochastic processes in electrochemistry. Anal. Chem. 2016, 88: 50175027.

- This paper thoroughly provides a very good introduction to the consequences of stochastic limitations in electrochemistry.

13 Kang S, Nieuwenhuis AbF, Mathwig K, Mampallil D, Lemay SG: Electrochemical singlemolecule detection in aqueous solution using self-aligned nanogap transducers. ACS Nano 2013: 10931-10937.

14 Mathwig K, Aartsma TJ, Canters GW, Lemay SG: Nanoscale methods for single-molecule electrochemistry. Annu. Rev. Anal. Chem. 2014, 7:383-404.

15 Kang S, Mathwig K, Mampallil D, Kostiuchenko Z, Lemay SG: Single-molecule electrochemistry in nanochannels: probing the time of first passage. Faraday Discuss. 2016, 193:41-50.

16 Xiong J, Chen Q, Edwards MA, White HS: Ion transport within high electric fields in nanogap electrochemical cells. ACS Nano 2015, 9:8520-8529.

17 Cao Z, Peng $\mathrm{Y}$, Voth $\mathrm{GA}$ : Ion transport through ultrathin electrolyte under applied voltages. J. Phys. Chem. B 2015, $119: 7516-7521$.

18 Fu K, Han D, Ma C, Bohn PW: Electrochemistry at single molecule occupancy in nanoporeconfined recessed ring-disk electrode arrays. Faraday Discuss. 2016, 193 :51-64.

19 Bae J, Yu Y, Mirkin MV: Recessed nanoelectrodes for nanogap voltammetry. ChemElectroChem 2016, $3: 2043-2047$.

20 Bae JH, Yu Y, Mirkin MV: Diffuse layer effect on electron-transfer kinetics measured by scanning electrochemical microscopy (SECM). J. Phys. Chem. Lett. 2017, $8: 1338-1342$.

21 Fu K, Han D, Ma C, Bohn PW: Ion selective redox cycling in zero-dimensional nanopore electrode arrays at low ionic strength. Nanoscale 2017, 9 :5164-5171.

22 Chen R, Najarian AM, Kurapati N, Balla RJ, Oleinick A, Svir I, Amatore C, McCreery RL, Amemiya S: Self-Inhibitory electron transfer of the $\mathrm{Co}$ (III)/Co(II)-complex redox couple at pristine carbon electrode. Anal. Chem., in press (Just Accepted Manuscript; DOI: 10.1021/acs.analchem.8b03023). 
23 One excellent volume of Faraday Discussions has been devoted to this topics (Faraday Discuss. 2016, 193); see a summary of the various topics discussed in: Crooks RM: Concluding remarks: single entity electrochemistry one step at a time. Faraday Discuss. 2016, 193: 533-547.

$24^{\circ}$ Grün F, Jardat M, Turq P, Amatore C: Relaxation of the electrical double layer after an electron transfer approached by Brownian dynamics simulation. J. Chem. Phys. 2004, 120: 9648-9655.

- This paper validated the use of Brownian dynamics simulations for investigating double layer relaxation after electron transfer as well as the importance of capacitive coupling.

25 Compare for example the anti-correlated current traces in Figures 1 and 4 in reference [13], in Figures 1d, 2 and 3 in reference [15] or in Figure 6 in: Kostiuchenko ZA, Glazer PJ, Mendes $E$, Lemay SG: Chemical physics of electroactive materials - the oft-overlooked faces of electrochemistry. Faraday Discuss. 2017, 199: 9-28.

$26^{\circ}$ Feldberg SW, Edwards MA: Current Response for a Single Redox Moiety Trapped in a Closed Generator-Collector System: The Role of Capacitive Coupling. Anal. Chem. 2015, 87: 3778-3783.

- This paper provides a first and elegant analytical approach to the problem discussed in this section based on analytical probabilities when the potential difference $\Delta E$ between the two electrodes is kept constant. Interestingly, it establishes that (i) the average time duration of a complete redox cycle (i.e., forward electron transfer followed by its backward one at the second electrode) is given by the classical result $L^{2} / D$ (where $L$ is the electrodes distance and $D$ is the diffusion coefficient), and (ii) that an average limiting current is reached at large $|\Delta E|$ values and whose expression is the same as for a generator-collector macrodevice.

27 Kätelhön E, Wolfrum B: Simulation-based investigations on noise characteristics of redoxcycling sensors. Phys. Status Solidi A 2012, 209: 881-884.

28 See e.g.: Balme S, Picaud F, Lepoitevin M, Bechelany M, Balanzatc E, Janota JM: Unexpected ionic transport behavior in hydrophobic and uncharged conical nanopores. Faraday Discuss. 2018, Advance Article (DOI: 10.1039/c8fd00008e).

29 Nazmutdinov RR, Bronshtein MD, Zinkicheva TT, Glukhov DV: Modeling of electron transfer across electrochemical interfaces: State-of-the art and challenges for quantum and computational chemistry. Int. J. Quantum Chem. 2016, 116: 189-201.

30 Futera Z, Sodeyama K, Burda JV, Einaga Y, Tateyama Y: A double-QM/MM method for investigating donor-acceptor electron-transfer reactions in solution. Phys. Chem. Chem. Phys. 2014, 16: 19530-19539.

- These two papers propose interesting elements for possible solutions to the problems discussed in the text.

31 For an early review, see e.g.: Silvester DS, Compton RG: Electrochemistry in room temperature ionic liquids: a review and some possible applications. Zeitschrift für Physikalische Chemie 2006, 220: 1247-1274.

32 Simon P, Gogotsi Y: Materials for electrochemical capacitors. Nat. Mater. 2008, 7: 845854. 
33 Bodin C, Mourad E, Zigah D, Le Vot S, Freunberger SA, Favier F, Fontaine O: Biredox ionic liquids: new opportunities toward high performance supercapacitors. Faraday Discuss. 2018, 206: 393-404.

34 Hayes R, Warr GG, Atkin R: Structure and Nanostructure in lonic Liquids. Chem. Rev. 2015, 115: 6357-6426.

35 Gehrke S, von Domaros M, Clark R, Holloczki O, Brehm M, Welton T, Luzare A, Kirchner B: Structure and lifetimes in ionic liquids and their mixtures. Faraday Discuss. 2018, 206: 219245.

36 Russina O, Triolo A: New experimental evidence supporting the mesoscopic segregation model in room temperature ionic liquids. Faraday Discuss. 2012, 154: 97-109.

37 Reichert P, Kjær KS, c van Driel TB, Mars J, Ochsmann JW, Pontoni D, Deutsch M, Nielsen $M D$, Mezger $M$ : Molecular scale structure and dynamics at an ionic liquid/electrode interface. Faraday Discuss. 2018, 206: 141-157.

$38^{\circ}$ Kakiuchi T, Nishi N: Ionic Liquid | Water Interface: A New Electrified System for Electrochemistry. Electrochemistry, 2006, 74: 942-948.

- Extremely important paper showing that double layers in RTILs have large inertial time constants.

39 See, e.g.: Evans RG, Klymenko OV, Price PD, Davies SG, Hardacre C, Compton RG: A comparative electrochemical study of diffusion in room temperature ionic liquid solvents versus acetonitrile. ChemPhysChem 2005, 6:526-33.

40 Lin C, Compton RG: Understanding mass transport influenced electrocatalysis at the nanoscale via numerical simulation. Current Opinion in Electrochemistry 2018, in press (doi.org/10.1016/j.coelec.2018.08.001).

41 See, e.g.: Buzzeo MC, Klymenko OV, Wadhawan JD, Hardacre C, Seddon KR, Compton RG: Voltammetry of Oxygen in the Room-Temperature lonic Liquids 1-Ethyl-3methylimidazolium Bis((trifluoromethyl)sulfonyl)imide and Hexyltriethylammonium Bis((trifluoromethyl)sulfonyl)imide: One-Electron Reduction To Form Superoxide. Steady-State and Transient Behavior in the Same Cyclic Voltammogram Resulting from Widely Different Diffusion Coefficients of Oxygen and Superoxide. J. Phys. Chem. A 2003, 107: 8872-8878.

42 Lu H, Nordholm S, Woodward CE, Forsman J: lonic liquid interface at an electrode : Simulations of electrochemical properties using an asymmetric restricted primitive model. J. Phys. Condens. Matter 2018, 30: 074004.

$43^{\circ}$ Chen M, Goodwin ZAH, Feng G, Kornyshev AA: On the temperature dependence of the double layer capacitance of ionic liquids. J. Electroanal. Chem. 2018, 819: 347-358.

- Publication evidencing the great difference between double layers in RTILs and in classical electrolytes

44 Savéant JM: Elements of molecular and biomolecular electrochemistry: an electrochemical approach to electron transfer chemistry. Wiley-Blackwell 2006.

45 Rudolph M, Reddy DP, Feldberg SW: A simulator for cyclic voltammetric responses. Anal. Chem. 1994, 66: 589A-600A. 
46 Rudolph M: Digital simulations on unequally spaced grids: Part 2. Using the box method by discretization on a transformed equally spaced grid. J. Electroanal. Chem. 2003, 543: 23-39.

47 Amatore C, Klymenko O, Svir, I: A new strategy for simulation of electrochemical mechanisms involving acute reaction fronts in solution: Principle. Electrochem. Commun. 2010, 12: 1170-1173.

48 Amatore C, Klymenko O, Svir, I: A new strategy for simulation of electrochemical mechanisms involving acute reaction fronts in solution: Application to model mechanisms. Electrochem. Commun. 2010, 12: 1165-1169.

49 See: www.kissagroup.com

$50^{\circ}$ See e.g. a typical example of such complexity in: Klymenko O, Buriez O, Labbe E, Zhan DP, Rondinini S, Tian ZQ, Amatore C: Uncovering a Missing Link between Molecular Electrochemistry and Electrocatalysis: Mechanism of Benzyl Chloride Reduction at Silver Cathodes. ChemElectroChem 2014, 1: 227-240.

- Publication demonstrating the importance of cross-linking electrochemical data with local spectroscopy (SERS) and DFT to establish the crucial importance of surface catalysis even when CVs look perfectly diffusional.

51 Klymenko OV, Svir I, Amatore C: Molecular Electrochemistry and Electrocatalysis: A Dynamic View. Molecular Physics 2014, 112: 1273-1283.

52 Sun T, Wang D, Mirkin MV: Electrochemistry at a single nanoparticle: from bipolar regime to tunnelling. Faraday Discuss. 2018, in press (DOI: 10.1039/c8fd00041g).

53 Amatore C, Savéant JM, Tessier D: Charge Transfer at Partially Blocked Surfaces. A Model for the Case of Microscopic Active and Inactive Sites. J. Electroanal. Chem. 1983, 147: 3951.

54 Sliusarenko O, Oleinick A, Svir I, Amatore C: Validating a Central Approximation in Theories of Regular Electrode Electrochemical Arrays of Various Common Geometries. Electroanalysis 2015, 27: 980-991.

55 Sliusarenko O, Oleinick A, Svir I, Amatore C: Development and Validation of an Analytical Model for Predicting Chronoamperometric Responses of Random Arrays of Micro- and Nanodisk Electrodes. ChemElectroChem 2015, 2: 1279-1291.

$56^{\circ \bullet}$ Sliusarenko O, Oleinick A, Svir I, Amatore C: Importance of Stochastic Limitations in Electrochemistry at Arrays of Nanoelectrodes Functionalized by Redox Self-Assembled Monolayers. Russian J. Electrochem. 2017, 53: 1019-1028.

- This publication demonstrates the consequences of reducing the number of redox mediators attached to nanoelectrodes

57 Nadjo L, Savéant JM, Tessier D: Convolution potential sweep voltammetry.3. Effect of sweep rate cyclic voltammetry. J. Electroanal. Chem. 1974, 52: 403-412.

$58^{\circ}$ Charoen-amornkitt $\mathrm{P}$, Suzuki T, Tsushima S: Ohmic resistance and constant phase element effects on cyclic voltammograms using a combined model of mass transport and equivalent circuits. Electrochim. Acta 2017, 258: 433-441.

- Publication demonstrating that the effect of CPE on CVs cannot be rationalized by a classical RC circuit. However, the theoretical approach selected by the authors is limited to mechanisms that can be described by linear equations. 
59 Aoki KJ, Chen J: Effects of the dipolar double layer on elemental electrode processes at micro- and macro-interfaces. Faraday Discuss. 2018, in press (DOI: 10.1039/C7FD00212B).

60 See e.g.: Hirschorn B, Orazem ME, Tribollet B, Vivier V, Frateur I, Musiani M: Determination of effective capacitance and film thickness from constant phase-element parameters. Electrochim. Acta 2010, 55: 6218-6227. 